\title{
BMJ Open Efficacy of the iDBT-Pain skills training intervention to reduce emotional dysregulation and pain intensity in people with chronic pain: protocol for a single-case experimental design with multiple baselines
}

\author{
Nell Norman-Nott, ${ }^{1,2}$ Chelsey Wilks, ${ }^{3}$ Negin Hesam-Shariati (D) ,1,2 \\ Jessica Schroeder, ${ }^{4}$ Jina Suh, ${ }^{4,5}$ Mary Czerwinski, ${ }^{5}$ Sylvia Maria Gustin ${ }^{1,2}$
}

To cite: Norman-Nott N, Wilks C, Hesam-Shariati N, et al. Efficacy of the iDBT-Pain skills training intervention to reduce emotional dysregulation and pain intensity in people with chronic pain: protocol for a single-case experimental design with multiple baselines. BMJ Open 2021;11:e041745. doi:10.1136/ bmjopen-2020-041745

- Prepublication history for this paper is available online. To view these files, please visit the journal online (http://dx.doi org/10.1136/bmjopen-2020041745).

Received 16 June 2020 Revised 08 March 2021 Accepted 09 March 2021

Check for updates

(C) Author(s) (or their employer(s)) 2021. Re-use permitted under CC BY-NC. No commercial re-use. See rights and permissions. Published by BMJ.

For numbered affiliations see end of article.

Correspondence to Dr Sylvia Maria Gustin; s.gustin@unsw.edu.au

\section{ABSTRACT}

Introduction Difficulties in emotional regulation are key to the development and maintenance of chronic pain. Recent evidence shows internet-delivered dialectic behaviour therapy (iDBT) skills training can reduce emotional dysregulation and pain intensity. However, further studies are needed to provide more definitive evidence regarding the efficacy of iDBT skills training in the chronic pain population.

Methods and analysis A single-case experimental design (SCED) with multiple baselines will be used to examine the efficacy of a 4-week iDBT-Pain skills training intervention (iDBT-Pain intervention) to reduce emotional dysregulation and pain intensity in individuals with chronic pain. The iDBT-Pain intervention encompasses two components: (1) iDBT-Pain skills training sessions (iDBT-Pain sessions) and (2) the iDBT-Pain skills training web application (iDBT-Pain app). Three individuals with chronic pain will be recruited and randomly allocated to different baseline phases (5, 9 or 12 days). Following the baseline phase, participants will receive six 60-90 min iDBT-Pain sessions approximately 4 or 5 days apart, delivered by a psychologist via Zoom. To reinforce learnings from the iDBT-Pain sessions, participants will have unlimited use of the iDBT-Pain app. A 7-day follow-up phase (maintenance) will follow the intervention, whereby the iDBT-Pain sessions cease but the iDBT-Pain app is accessible. Emotional regulation, as the primary outcome measure, will be assessed using the Difficulties in Emotion Regulation Scale. Pain intensity, as the secondary outcome measure, will be assessed using a visual analogue scale. Generalisation measures will assess psychological state factors (depression, anxiety and coping behaviour), alongside sleep quality, well-being and harm avoidance. SCEDs are increasingly considered effective designs for internet-delivered psychological interventions because SCED enables the investigation of interindividual variability in a heterogeneous population such as chronic pain.

Ethics and dissemination This trial was approved by the University of New South Wales (HC200199). Results will be published in peer-reviewed journals.
Strengths and limitations of this study

- Multiple baseline single-case experimental designs (SCEDs) provide rich data about individual participant responses to an intervention that is useful to inform evidence-based clinical practice.

- SCED studies are a viable alternative when the recruitment of a large cohort needed for a randomised clinical trial is not feasible.

- SCED methodology accommodates for group heterogeneity, typical within a chronic pain population.

- Between-participant replications in a multiple baseline SCED contribute to the external validity of the study.

- Evaluating the efficacy of the treatment relies on all participants completing the three phases of the study, thus, if there is any dropout the ability to demonstrate treatment effects will be limited.

Trial registration number ACTRN12620000604909.

\section{BACKGROUND}

Chronic pain, defined as pain lasting longer than 3 or 6 months, ${ }^{1}$ is widely acknowledged as a global issue affecting around one in five adults in the general population. ${ }^{23}$ While the focus for research on chronic pain has historically been on its sensory components, affective factors are now commonly understood in the literature to also be related. ${ }^{2}$ Specifically, emotional states play a key role in the development, maintenance, assessment and treatment of chronic pain. ${ }^{4}$ Indeed, research links chronic pain to major difficulties in emotional regulation, defined as an individual's ability to control and manage emotional states and expression, including the type of emotions expressed alongside when and 
how these emotions occur. ${ }^{3}$ For example, individuals frequently experience increased fear and worry about themselves and their pain, but also more broadly about family, their social life and the future. ${ }^{5}$ Accordingly, people with chronic pain cannot define and pursue meaningful goals because they are not able to regulate negative emotions, which results in a vicious cycle of chronic disability, decreased quality of life and emotional suffering. ${ }^{6}$ One chronic pain sufferer (a prior participant from a study conducted by the current research group) described how the emotions associated with pain can be overwhelming:

I am afraid of my pain constantly because I know how bad it can get. That makes me worry and think about it all the time which causes anger and sadness because I am wasting my life. I don't know how to deal with it. But sometimes the emotions are harder to deal with than the pain itself. That stresses me out.

In addition to exploring the lived experience of chronic pain, our group found evidence to support a neurobiological link between emotional dysregulation and chronic pain. Specifically, the stress associated with the experience of chronic pain decreases glutamate, an excitatory neurotransmitter, in the medial prefrontal cortex, resulting in emotional dysregulation. ${ }^{78}$ These findings align with animal models of chronic pain, whereby stress mechanisms such as increased levels of glucocorticoids with the occurrence of chronic pain lead to a decline in glutamate in the medial prefrontal cortex resulting in a change in patterns of emotional behaviour. ${ }^{910}$ Collectively, these findings demonstrate that neurobiological processes underpin emotional dysregulation in chronic pain.

Cognitive-behavioural therapy (CBT) is commonly used to treat chronic pain and negative emotional comorbidities such as mood disorders. ${ }^{11}$ However, a Cochrane review $^{12}$ reveals that $\mathrm{CBT}$ has no effect on pain intensity or mood when compared with active controls. CBT has a small-or at best, moderate-effect on pain intensity and mood immediately after therapy when compared with treatment as usual or waiting list, and only a small effect on mood is sustained at 6 months of follow-up. ${ }^{12}$ Naylor and colleagues ${ }^{6}$ explain these findings by proposing that CBT may be limited to treat chronic pain because it does not substantially address the difficulties in emotional regulation commonly associated with chronic pain. Indeed, Lumley and colleagues ${ }^{4}$ suggested that emotional factors are intrinsically interwoven with chronic pain, thus incorporating intensive skills training for emotional regulation into the cognitive-behavioural model for pain may enhance treatment effectiveness.

Dialectical behaviour therapy (DBT) is a specific type of CBT developed by Marsha Linehan, ${ }^{13}$ originally for highly suicidal individuals with high emotion dysregulation to learn skills to more effectively manage their emotions. DBT helps people cope with painful, fearful, worrisome, anxious and negative thoughts and emotions as well as rumination, ${ }^{14-16}$ and has been shown to alleviate negative emotional symptoms in individuals presenting with high emotional states. ${ }^{17} 18$ In the chronic pain context, Linton ${ }^{19}$ demonstrated in a case study with a 52-year-old woman with chronic musculoskeletal pain that DBT lowers emotional dysregulation and pain intensity. Moreover, in a pilot study with chronic pain sufferers $(n=6)$, an 8-week DBT programme improved pain intensity and symptoms of anxiety and depression. ${ }^{20}$ Despite the success of these preliminary studies, to our knowledge, no other researchers have investigated DBT within the chronic pain population. Additionally, despite technologically delivered therapies having the advantage of delivery to remote communities and to those with limited access to face-to-face treatment, ${ }^{21}{ }^{22}$ no known study has investigated online DBT treatment with a chronic pain population, who frequently suffer from restricted mobility associated with chronic pain. ${ }^{23}$ Indeed, it was recently suggested that individuals with chronic pain should be rapidly introduced to internet-delivered treatment during the COVID-19 outbreak because treatment centres across the world have had to shut their doors, making chronic pain treatment largely inaccessible unless delivered online. ${ }^{24}$

The current trial will use a single-case experimental design (SCED) with multiple baselines to evaluate the efficacy of a 4-week internet-delivered DBT skills training intervention (iDBT-Pain intervention) for chronic pain sufferers to decrease emotional dysregulation and pain intensity. Given that chronic pain is consistently associated with a poor quality of life and can be debilitating for many sufferers, this trial may present a new viable therapy for chronic pain. Moreover, by having the DBT skills training online it is more accessible to those in remote communities, to those with restricted mobility, and more broadly it adds to the knowledge of internet-delivered therapies at a time when online is increasingly necessary to deliver treatment due to COVID-19.

\section{Study objectives}

The primary objective is to evaluate the efficacy of the iDBT-Pain intervention delivered over 4 weeks, to lower emotional dysregulation in individuals with chronic pain. The secondary objective is to investigate the efficacy of the iDBT-Pain intervention to reduce pain intensity in chronic pain sufferers. We will also determine whether the iDBT-Pain intervention improves psychological state factors (depression, anxiety and coping behaviour) alongside sleep quality, well-being and harm avoidance.

\section{METHODS}

\section{Study design}

The efficacy of the iDBT-Pain intervention will be evaluated using a randomised, concurrent SCED with multiple baselines across participants. The design consists of $\mathrm{AB}+$ follow-up, where $\mathrm{A}$ refers to a baseline phase, $\mathrm{B}$ is the intervention phase and the follow-up is a maintenance phase. 
In the SCED approach, participants are repeatedly assessed on at least one dependent variable (in this case, emotional dysregulation and pain intensity) across the phases of the study. SCED guidelines state that at least five reported measures of the dependent variables in each phase are taken across a minimum of three participants to evaluate the effectiveness of an intervention. ${ }^{25}$ The construction of this SCED trial and the reporting of the results will be in accordance with the Single-Case Reporting Guidelines in Behavioural Intervention. ${ }^{26}$

SCEDs are increasingly being considered to evaluate the efficacy of psychological interventions for chronic pain because SCEDs accommodate for heterogeneity and large interindividual variability in a population, as is typical in those with chronic pain. ${ }^{27}$ SCED allows investigation of the intervention effects at an individual level to reveal deep insights about specific subjects' response to treatment and provide rich data about the sequence of changes during treatment. ${ }^{27} 28$ These insights can inform interventions for specific psychological changes associated with chronic pain and can help bridge the scientistclinician gap to inform more individualised treatment plans. ${ }^{29}$ Moreover, some researchers have concluded that a highly controlled, randomised, multiple baseline SCED may be on par with a randomised controlled trial, which have commonly been considered the gold standard to determine treatment efficacy in a clinical trial. ${ }^{30}$

The power of SCED studies derives not from the number of participants, but from the number of repeated measures across the phases of the study. ${ }^{31}$ Multiple measurement timepoints enable experimental control because the instance a change occurs in the outcome measure for a particular participant during treatment, preliminary efficacy of the intervention is demonstrated. ${ }^{32}$ Replication of the experimental effect across participants, also called direct replication, is the foundation for internal validity in SCED studies, ${ }^{31}$ with three replications minimum to demonstrate the efficacy of a treatment. ${ }^{25}$

Scientific credibility is increased in SCED trials by incorporating randomisation in the experimental design. ${ }^{33}$ For example, in the current study, participants will be randomly assigned to different durations for the baseline phase. By adding in this element of randomisation, the internal validity of a SCED study is enhanced because there is increased control over confounding factors such as the setting, the participants and the time. ${ }^{27}$ While a SCED trial may have limited generality due to the number of participants, synthesising results from several trials assessing individual responses to treatment can enhance external validity. ${ }^{34}$ Moreover, the incorporation of generalisation measures in a SCED study can reveal whether the treatment effects beyond the primary and secondary outcome measures to influence relevant factors that are not directly targeted by the treatment. ${ }^{31}$

\section{Procedure}

Using a simple randomisation method, ${ }^{35}$ three participants will be assigned to one of three different tiers in

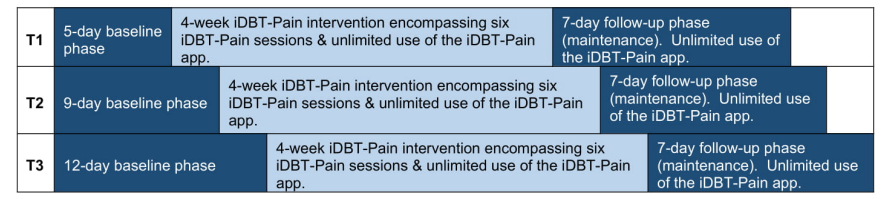

Figure 1 Study procedure. Participants will be randomly assigned to one of the three tiers. The baseline phase will be followed by the iDBT-Pain intervention. The iDBT-Pain intervention encompasses two components: (1) iDBT-Pain sessions and (2) the iDBT-Pain app. During the intervention phase participants will receive six 60-90 min iDBT-Pain sessions delivered by a psychologist via Zoom, 4-5 days apart, across 4 weeks. To supplement and reinforce the learnings from the iDBT-Pain sessions, participants will have unlimited use of the iDBT-Pain app. The 4-week iDBT-Pain intervention will be followed by a 7-day follow-up phase (maintenance) where participants will have no contact with the psychologist but will continue to access the iDBT-Pain app. iDBT, internet-delivered dialectic behaviour therapy; iDBT-Pain app, iDBT-Pain skills training website application; iDBT-Pain intervention, iDBT-Pain skills training intervention; iDBT-Pain sessions, iDBT-Pain skills training sessions; T, tier.

the baseline phase. The three baseline tiers will differ in terms of duration, allowing the study to achieve SCED multiple baseline randomisation criteria. ${ }^{26}$ The first tier will include a 5-day baseline to allow adequate time for stabilisation. ${ }^{36}$ The subsequent two baseline tiers will be 9 days (tier 2) and 12 days (tier 3 ). In all three baseline tiers, participants will be measured daily for the dependent variables (in this case, emotional regulation and pain intensity).

The baseline phase will start concurrently for all three tiers, and on conclusion will be immediately followed by the iDBT-Pain intervention. The iDBT-Pain intervention encompasses two components: (1) iDBT-Pain skills training sessions (iDBT-Pain sessions) and (2) the iDBTPain skills training web application (iDBT-Pain app). During the intervention phase participants will receive six 60-90 min iDBT-Pain sessions delivered by a psychologist via Zoom, 4-5 days apart, across 4 weeks. To supplement and reinforce the learnings from the iDBT-Pain sessions, participants will have unlimited use of the iDBTPain app. The 4-week intervention phase will be immediately followed by a 7-day follow-up phase to maintain the skills learnt during the iDBT-Pain intervention. During the follow-up (maintenance) phase, the iDBT-Pain sessions with the psychologist will cease but participants will continue to have access to the iDBT-Pain app. The primary and secondary outcome measures will continue to be assessed daily in the follow-up phase. For a summary of the study procedure, see figure 1 .

\section{Participants}

Three individuals with chronic pain will be recruited to the study. The following inclusion criteria must be met by all participants: (1) aged 18-80 years, (2) chronic pain for $\geq 6$ months, and (3) demonstrating an ability to use the visual analogue scale (VAS). Participants with all types of 
chronic pain will be accepted into the study. Participants' pharmacological and non-pharmacological treatment will be documented before, during and after intervention.

\section{Intervention}

A core component of evidence-based DBT practice is skills training on several key areas, such as emotional regulation and mindfulness, to help individuals more effectively regulate negative cognitions and emotions. ${ }^{37}$ Skills training equips an individual with the ability to identify the triggers that stimulate negative states and to apply coping skills to the sequence of thoughts, feelings and behaviours to circumvent adverse negative reactions and emotions. ${ }^{38}$ In the current study, DBT skills training is delivered to the participants in the iDBT-Pain intervention which encompasses two components: (1) iDBT-Pain sessions and (2) the iDBT-Pain app.

\section{iDBT-Pain sessions}

Six iDBT-Pain sessions will be delivered individually by a psychologist to each participant over the videoconferencing platform Zoom at agreed times, approximately 4-5 days apart, during a 4-week period. DBT has a modular structure, designed so that components can be dropped in and pulled out according to client needs. ${ }^{13}$ This modularity makes DBT ideal for delivery in a digital environment because content may be divided up and delivered as separate necessary skills to train. ${ }^{39}$ The six iDBT-Pain sessions are based on the internet-delivered protocol developed by Wilks and colleagues, ${ }^{22}$ but modified for the chronic pain population (figure 2). They will address the following three core DBT skills that are most relevant for a chronic pain population: mindfulness (two sessions), emotional regulation (three sessions) and distress tolerance (one session). The iDBT-Pain sessions will be conducted by an Australian Health Practitioner Regulation Agency registered psychologist.

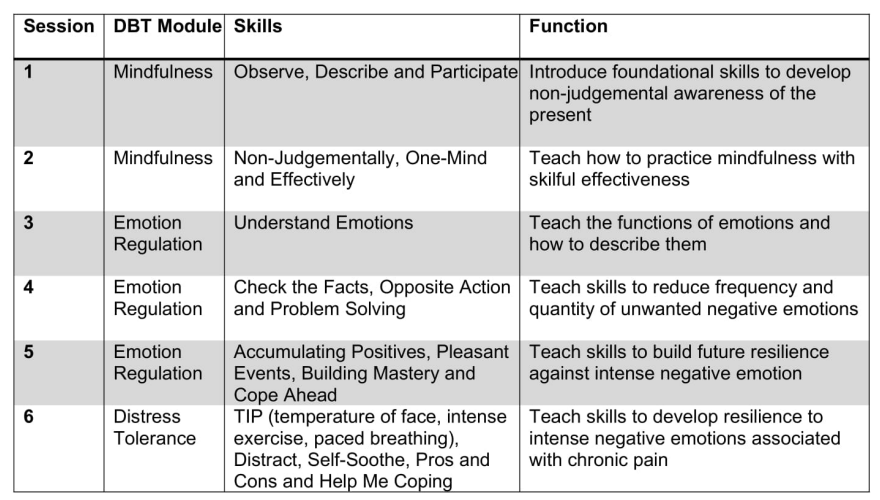

Figure 2 Outline for the iDBT-Pain sessions to be delivered by a psychologist online via Zoom approximately every 4-5 days across 4 weeks. The iDBT-Pain sessions are adapted from Wilks and colleagues, ${ }^{22}$ and modified for the chronic pain population. iDBT-Pain sessions, internetdelivered dialectic behaviour therapy-Pain skills training sessions.

\section{iDBT-Pain app}

Successful treatment for emotional dysregulation with DBT is associated with a generalisation of skills to everyday life which is facilitated by regular homework encompassing videos and skills-based apps. ${ }^{13}$ Accordingly, during the first iDBT-Pain session participants will be shown how to work through the iDBT-Pain app. The iDBT-Pain app is based on an app developed by Wilks and colleagues, ${ }^{40}$ but for the current trial, it has been modified for the chronic pain population. At each iDBT-Pain session, participants will be asked to focus on a specific DBT skill for that week (eg, mindfulness) by watching the videos and completing the skills training tasks before the next iDBT-Pain session. Thus, completing tasks in the app will be homework to help reinforce the skills learnt during the iDBT-Pain sessions. Participants will be encouraged by the researchers to participate in the modules on the iDBT-Pain app every day.

\section{Outcome measures}

\section{Primary outcome measure}

The primary outcome measure is emotional regulation which will be measured using the Difficulties in Emotion Regulation Scale ${ }^{41}$ (DERS). The 18-item version will be used to assess participants' levels of emotional regulation. Items are rated on a scale of 1 ('almost never') to 5 ('almost always') with higher scores indicating increased difficulty in emotion regulation. Previous research supports the use of the DERS-18 in a chronic pain population. ${ }^{42}$ The DERS will be measured on every evening of the baseline and follow-up phase as well as after each of the six iDBT-Pain sessions.

\section{Secondary outcome measure}

The secondary outcome measure is pain intensity which will be measured using a VAS. A VAS for pain intensity encompasses a $10 \mathrm{~cm}$ horizontal line which ranges from 'no pain' at one end to 'worst imaginable pain' at the other end. Participants will be asked and are required to rate their average pain intensity experienced across three specified time ranges every day by marking the line accordingly. Participants will be asked at 12 midday to mark their average pain intensity since waking. The second time period is between 12 midday and 18:00, when participants will be asked to again rate their average level of pain intensity. The final measure of pain intensity in a day is after 18:00 but before going to sleep, when each participant will once again denote the point that best indicates their average pain intensity experienced. A daily average will be calculated to denote the pain intensity experienced for each participant on a given day. For any missing data points, the participants' average will be taken from the pain intensity ratings that are observed that day. For those participants who can complete a VAS, extensive evidence supports its validity and reliability to evaluate pain intensity. ${ }^{43}$ A VAS will be completed on paper as a daily pain diary during all three phases of 
the current study (baseline, iDBT-Pain intervention and follow-up) and across all three tiers of the study.

To address any questions about the DERS ${ }^{41}$ and the VAS measures, and to enable compliance in completing the measures, the study researchers will be in daily contact with participants.

\section{Generalisation measures}

Generalisation measures will be used to evaluate whether there are any additional relevant changes to participants' behaviour that may be attributed to the intervention, beyond the primary and secondary outcomes. Thus, generalisation measures bolster external validity by showing that an intervention can generalise outside of the studies' target behaviours. ${ }^{44}$ In the current study, generalisation measures will include psychological questionnaires. The psychological assessment will be administered at two timepoints: (1) prior to the baseline phase and (2) following completion of the follow-up (maintenance) phase.

\section{Psychological questionnaires}

Participants will complete the COMPAS-W Scale of Wellbeing, ${ }^{45}$ the State Anxiety Inventory (SAI) ${ }^{46}$ the Beck Depression Inventory (BDI), ${ }^{47}$ the Medical Outcomes Study Sleep Scale (MOS-SS), ${ }^{48}$ the Dialectic Behaviour Therapy Ways of Coping Checklist (DBT-WCCL) ${ }^{18}$ and the Temperament and Character Inventory Harm Avoidance Scale (TCI-HA). ${ }^{49}$ All these measures are self-report questionnaires. Psychological and subjective well-being will be measured with the COMPAS-W, ${ }^{45}$ a 26-item scale split across six subscales of composure, own worth, mastery, positivity, achievement and satisfaction. Higher scores will denote higher levels of well-being. State anxiety will be measured with the 20-item $\mathrm{SAI}^{46}$ with higher scores indicating greater anxiety. Depressive symptoms will be assessed with the 21-item $\mathrm{BDI}^{47}$ with a higher score indicating more severe depressive symptoms. Sleep will be measured via the 12-item MOS-SS ${ }^{48}$ to evaluate sleep initiation, maintenance, respiratory problems, quantity, perceived adequacy and somnolence. The DBT-WCCL $^{18}$ is a 59 -item measure to assess the types of coping behaviour that are employed. Higher scores on the general dysfunction and blaming others scales indicate lower ability to cope and higher scores on the skills use scale indicate greater usage of DBT skills for coping. Lastly, the TCI-HA ${ }^{49}$ is a 35 -item measure to assess levels of the temperament harm avoidance with scores summed and converted to a percentile from $0 \%$ to $100 \%$. Higher scores indicate higher levels of harm avoidance.

\section{Feasibility, satisfaction and experience measures}

Following the completion of the 4-week iDBT-Pain intervention, participants will be asked to complete the Usefulness, Satisfaction, and Ease of Use questionnaire ${ }^{50}$ to measure feasibility. The Patient Global Impression of Change $^{51}$ scale will be used to measure the perceived change in emotional regulation and pain intensity, respectively. An unstructured interview will be conducted with each participant after completion of the intervention to further assess their perception of safety and feasibility as well as experience with the iDBT-Pain sessions and iDBT-Pain app.

\section{Data analysis}

Primary and secondary outcomes analysis

SCED analysis is primarily reliant on visual inspection to describe the features of the data; however, statistical analysis extends understanding about SCED data to determine the strength of the relationship between the intervention and participants' behaviour. ${ }^{36}$ In the current study, the primary and secondary outcome measures will be separately analysed using both visual and statistical analyses.

\section{Visual analysis}

In accordance with typical visual analysis for SCED, a structured analysis will be used whereby individual participant data will be graphically represented as a line graph for each of the outcome variables. ${ }^{3152}$ The baseline phase in each tier will then be observed to determine a stable benchmark or control, with any changes observed in the data during the intervention and follow-up phases revealing any notable alteration to the outcome measures. Further, participant's data will be examined to determine whether any changes observed to the outcome measures of interest are reflected across participants. Accordingly, all data will be mapped and inspected using both a withinphase analysis and a between-phase analysis. A withinphase analysis means an evaluation of data patterns across participants in a single phase, and a between-phase analysis means a comparison across adjacent phases for each participant. $^{52}$

To determine the extent of the change to the outcome variables in a SCED trial, it has been widely recommended that the following key factors are considered when interpreting the data: (A) level, (B) trend, (C) stability, and (D) overlap. ${ }^{534}$

The magnitude of behaviour change is the (A) level that is observed both within phase or across the tiers, and between phases for each participant. ${ }^{52}$ In the current study, to account for any possible latency in the intervention effects, the median values will be used to determine the change in level.

Lane and Gast define (B) trend as the directional pattern of the data over time. ${ }^{52}$ Trend will be estimated using the split-middle method with the trend line calculated for each phase of each tier. ${ }^{36}$ It is expected that in the baseline phase, the data points will progress in a zero-correlated direction, but in the intervention phase, a decelerating trend will be observed and will continue in the follow-up phase.

In SCED studies, (C) stability in the baseline phase is important to allow the investigation of the effect of an intervention in the subsequent phase. ${ }^{36}$ In line with current SCED practices, data points will be considered 
stable when approximately $80 \%$ of the values are within $25 \%$ of the median value. ${ }^{52}$

Finally, (D) overlap refers to the proportion of data in a phase that overlaps with data from a previous phase, with lesser overlap signifying a greater effect of the intervention and greater overlap suggesting lower effect of the intervention. ${ }^{55}$ Percentage of data points exceeding the median $\left(\mathrm{PEM}^{56}\right)$ of the baseline phase will be compared with the data points in the subsequent phases. PEM has an advantage over other overlap approaches because it is less likely to be influenced by autocorrelation when data points are captured in a successive time period. ${ }^{57}$

\section{Statistical analysis}

The current study will use Tau- $U$ to statistically analyse the overlap between baseline and intervention, and baseline and follow-up. Tau- $U$ will further be used to provide an effect size and weighted average across participants with CIs based on the number of data points. ${ }^{58}$ As a non-parametric test, Tau- $U$ is appropriate for small data sets, typical of SCED studies, because it does not require outcome data to conform to independence or normality assumptions. ${ }^{59}$ Notably, Tau- $U$ can approximate and control for an undesirable trend in the baseline phase to identify whether changes observed in the intervention phase are due to the treatment or a naturally occurring tendency. ${ }^{5960}$ For the current study, the following web application will be used to compute Tau- $U$ to analyse the data: http://www.singlecaseresearch.org/calculators/ tau-u ${ }^{61}$

\section{Generalisation measures analysis}

\section{Psychological questionnaires}

Total scores and subscores of the psychological questionnaires (COMPAS-W Scale, ${ }^{45}$ SAI ${ }^{46}$ BDI,${ }^{47}$ MOS-SS, ${ }^{62}$ TCI$\mathrm{HA}^{49}$ and DBT-WCCL ${ }^{18}$ ) will be computed for further analysis (see the Reliable change index section).

\section{Reliable change index}

The reliable change index (RCI) is used to evaluate whether an individual score changes over time to determine whether the difference between a premeasurement and postmeasurement is statistically and clinically significant. ${ }^{6364}$ In the current study, the generalisation measures (psychological questionnaires) will be evaluated with the RCI to investigate the reliability of the change from preintervention to postintervention.

$\mathrm{RCI}$ is a ratio of the actual observed difference by the standard error of the difference $\left(S E_{\text {diff }}\right)$ :

$$
\begin{gathered}
R C I=\frac{M_{\text {post }}-M_{\text {pre }}}{S E_{\text {diff }}} \\
S E_{\text {diff }}=S D \sqrt{2(1-r)}
\end{gathered}
$$

$S D$ is the standard deviation of the measurement and $r$ is the reliability coefficient of the measure.

\section{Patient and public involvement}

Multiple channels will be used to disseminate the research findings, including press releases, conferences, publications and communication, to consumer groups specifically chronic pain sufferers and associated stakeholders.

\section{DISCUSSION}

Emerging evidence has shown that negative emotional states play a key role in the development and maintenance of chronic pain. ${ }^{4}$ Preliminary findings in support of DBT to treat chronic pain and its comorbidities, such as anxiety and depressive symptoms, have been reported. ${ }^{19}{ }^{20}$ However, further studies are needed to provide more definitive evidence regarding the effectiveness of this intervention within a chronic pain population, specifically in relation to reducing emotional dysregulation alongside pain intensity. This paper details the study protocol for the evaluation of the iDBT-Pain intervention to improve emotional dysregulation, pain intensity and other psychological state and trait factors (anxiety, depression, coping behaviour, harm avoidance, well-being and sleep quality) in a chronic pain population.

This study will use a concurrent and randomised multiple baseline SCED methodology which is appropriate when investigating treatment effects within small or very heterogeneous populations, such as the chronic pain population. ${ }^{27}$ Moreover, a SCED design is more feasible and more appropriate than a large group-based study when only preliminary investigations have shown treatment success with DBT for chronic pain. ${ }^{31}$ Indeed, randomised multiple baseline SCED trials are argued to be powerful and effective designs because they capture rich data about the sequence of changes during treatment to help inform evidence-based practice. ${ }^{29}$

\section{ETHICS AND REGISTRATION}

This study protocol has been approved by the University of New South Wales Human Research Ethics Committee (approval number: HC200199). This protocol is registered through the Australian and New Zealand Clinical Trials Registry (registration number: ACTRN12620000604909). The anticipated date to start the trial is July 2020.

\section{Author affiliations}

${ }^{1}$ Centre for Pain IMPACT, Neuroscience Research Australia, Randwick, New South Wales, Australia

${ }^{2}$ School of Psychology, University of New South Wales, Sydney, New South Wales, Australia

${ }^{3}$ Department of Psychological Science, University of Missouri-St Louis, St Louis, Missouri, USA

${ }^{4}$ School of Computer Science and Engineering, University of Washington, Seattle, Washington, USA

${ }^{5}$ Microsoft Research, Redmond, Washington, USA

Acknowledgements We thank Jeremy Norman-Nott for his work to customise the iDBT-Pain app for use in this study. Thanks to Jeremy's work that the researchers have been able to tailor the app content for use within the chronic pain population.

Contributors NNN, CW, NHS and SMG designed the SCED protocol. NNN and SMG wrote the first draft of the manuscript. NNN, CW, JSc, JSu, MC and SMG developed the iDBT-Pain web app. All authors critically revised the manuscript and approved the final version. 
Funding This work was supported by the Rebecca L Cooper Medical Research Foundation.

Competing interests CW receives consulting fees from Mindstrong Health and Lyra Health. JSu reports other from Microsoft, outside the submitted work.

Patient and public involvement Patients and/or the public were not involved in the design, or conduct, or reporting, or dissemination plans of this research.

Patient consent for publication Not required.

Provenance and peer review Not commissioned; externally peer reviewed.

Open access This is an open access article distributed in accordance with the Creative Commons Attribution Non Commercial (CC BY-NC 4.0) license, which permits others to distribute, remix, adapt, build upon this work non-commercially, and license their derivative works on different terms, provided the original work is properly cited, appropriate credit is given, any changes made indicated, and the use is non-commercial. See: http://creativecommons.org/licenses/by-nc/4.0/.

ORCID iD

Negin Hesam-Shariati http://orcid.org/0000-0003-0829-7016

\section{REFERENCES}

1 Otis JD, Flor H, Turk DC. 2011) chronic pain: an integrated biobehavioral approach. Seattle: IASP press: Elsevier, 2013.

2 Gatchel RJ, Peng YB, Peters ML, et al. The biopsychosocial approach to chronic pain: scientific advances and future directions. Psychol Bull 2007;133:581.

3 Koechlin $\mathrm{H}$, Coakley R, Schechter $\mathrm{N}$, et al. The role of emotion regulation in chronic pain: a systematic literature review. $J$ Psychosom Res 2018;107:38-45.

4 Lumley MA, Cohen JL, Borszcz GS, et al. Pain and emotion: a biopsychosocial review of recent research. J Clin Psychol 2011;67:942-68.

5 Tang NKY, Salkovskis PM, Hodges A, et al. Chronic pain syndrome associated with health anxiety: a qualitative thematic comparison between pain patients with high and low health anxiety. $\mathrm{Br} J$ Clin Psychol 2009;48:1-20.

6 Naylor B, Boag S, Gustin SM. New evidence for a pain personality? A critical review of the last 120 years of pain and personality. Scand $J$ Pain 2017;17:58-67.

7 Kang D, McAuley JH, Kassem MS, et al. What does the grey matter decrease in the medial prefrontal cortex reflect in people with chronic pain? Eur J Pain 2019;23:203-219.

8 Naylor B, Hesam-Shariati N, McAuley JH, et al. Reduced glutamate in the medial prefrontal cortex is associated with emotional and cognitive dysregulation in people with chronic pain. Front Neurol 2019;10:1110.

9 Metz AE, Yau H-J, Centeno MV, et al. Morphological and functional reorganization of rat medial prefrontal cortex in neuropathic pain. Proc Natl Acad Sci U S A 2009;106:2423-8.

10 Fiore NT, Austin PJ. Are the emergence of affective disturbances in neuropathic pain states contingent on supraspinal neuroinflammation? Brain Behav Immun 2016;56:397-411.

11 Ehde DM, Dillworth TM, Turner JA. Cognitive-Behavioral therapy for individuals with chronic pain: efficacy, innovations, and directions for research. Am Psychol 2014;69:153.

12 Williams ACdeC, Eccleston C, Morley S, et al. Psychological therapies for the management of chronic pain (excluding headache) in adults. Cochrane Database of Systematic Reviews 2012;19.

13 Linehan M. Dbt skills training manual. Second. New York: Guilford Press, 2015.

14 Chapman A, Gratz K, Tull M. The dialectical behavior therapy skills workbook for anxiety: breaking free from worry, panic, PTSD, and other anxiety symptoms. Oakland, California: New Harbinger Publications, 2011

15 Leeuw M, Goossens MEJB, Linton SJ, et al. The fear-avoidance model of musculoskeletal pain: current state of scientific evidence. $J$ Behav Med 2007:30:77-94.

16 Marra T. Depressed and anxious: the dialectical behavior therapy workbook for overcoming depression and anxiety. Oakland, California: New Harbinger Publications, 2004.

17 Lynch TR, Morse JQ, Mendelson T, et al. Dialectical behavior therapy for depressed older adults: a randomized pilot study. Am J Geriatr Psychiatry 2003;11:33-45.

18 Neacsiu AD, Rizvi SL, Vitaliano PP, et al. The dialectical behavior therapy ways of coping checklist: development and psychometric properties. J Clin Psychol 2010;66:563-82.
19 Linton SJ. Applying dialectical behavior therapy to chronic pain: a case study. Scand J Pain 2010:1:50-4.

20 Sysko H, Thorkelson G, Szigethy E. P-001 dialectical behavior therapy for chronic pain in gastrointestinal disorders: a pilot study. Inflamm Bowel Dis 2016;22:S9.

21 Karyotaki E, Riper H, Twisk J, et al. Efficacy of self-guided Internetbased cognitive behavioral therapy in the treatment of depressive symptoms: a meta-analysis of individual participant data. JAMA Psychiatry 2017;74:351-9.

22 Wilks CR, Lungu A, Ang SY, et al. A randomized controlled trial of an Internet delivered dialectical behavior therapy skills training for suicidal and heavy episodic drinkers. J Affect Disord 2018;232:219-28.

23 Brennan F, Carr DB, Cousins M. Pain management: a fundamental human right. Anesth Analg 2007;105:205-21.

24 Eccleston C, Blyth FM, Dear BF, et al. Managing patients with chronic pain during the COVID-19 outbreak: considerations for the rapid introduction of remotely supported (eHealth) pain management services. Pain 2020;161:889-93.

25 Kratochwill TR, Hitchcock JH, Horner RH, et al. Single-case intervention research design standards. Remedial and Special Education 2013;34:26-38.

26 Tate RL, Perdices M, Rosenkoetter U, et al. The Single-Case reporting guideline in behavioural interventions (SCRIBE) 2016 statement. Archives of Scientific Psychology 2016;4:1-9.

27 Onghena P, Edgington ES. Customization of pain treatments: singlecase design and analysis. Clin J Pain 2005;21:56-68.

28 Byiers BJ, Reichle J, Symons FJ. Single-subject experimental design for evidence-based practice. Am J Speech Lang Pathol 2012;21:397-414.

29 Vlaeyen JWS, Crombez G. Behavioral conceptualization and treatment of chronic pain. Annu Rev Clin Psychol 2020;16:187-212.

30 Romeiser Logan L, Hickman RR, Harris SR, et al. Single-subject research design: recommendations for levels of evidence and quality rating. Dev Med Child Neurol 2008;50:99-103.

31 Krasny-Pacini A, Evans J. Single-case experimental designs to assess intervention effectiveness in rehabilitation: a practical guide. Ann Phys Rehabil Med 2018;61:164-79.

32 Dallery J, Cassidy RN, Raiff BR. Single-case experimental designs to evaluate novel technology-based health interventions. J Med Internet Res 2013:15:e22.

33 Kratochwill TR, Levin JR. Enhancing the scientific credibility of single-case intervention research: randomization to the rescue. Psychol Methods 2010;15:124-44.

34 Vlaeyen JWS, Wicksell RK, Simons LE, et al. From Boulder to Stockholm in 70 years: single case experimental designs in clinical research. Psychol Rec 2020;70:659-70.

35 Altman DG, Bland JM. How to randomise. BMJ 1999;319:703-4.

36 Tate RL, Perdices M. Single-Case experimental designs for clinical research and Neurorehabiitation settings. New York: Routledge, 2019.

37 Neacsiu AD, Eberle JW, Kramer R, et al. Dialectical behavior therapy skills for transdiagnostic emotion dysregulation: a pilot randomized controlled trial. Behav Res Ther 2014;59:40-51.

38 Linehan MM. DBT skills training manual. 2 ed. New York: The Guildford Press, 2015.

39 Lungu A. Computerized trans-diagnostic dialectical behavior therapy skills trainingfor emotion dysregulation [Doctoral dissertation, University of Washington], 2015. Available: https://digital.lib. washington.edu/researchworks/bitstream/handle/1773/34148/ Lungu_washington_0250E_14912.pdf?sequence $=1$ \&isAllowed $=y$

40 Wilks C, Czerwinski M, Rowan K, et al. Machine learning for precise targeting of a mobile dialectical behaviortherapy skills training application. [Conference Session]. CHI '17: ACM CHI Conference on Human Factors in Computing Systems, Denver, Colorado, UnitedStates, 2017.

41 Victor SE, Klonsky ED. Validation of a brief version of the difficulties in emotion regulation scale (DERS-18) in five samples. $J$ Psychopathol Behav Assess 2016;38:582-9.

42 Lutz J, Gross RT, Vargovich AM. Difficulties in emotion regulation and chronic pain-related disability and opioid misuse. Addict Behav 2018;87:200-5.

43 Hawker GA, Mian S, Kendzerska T, et al. Measures of adult pain: visual analog scale for pain (vas pain), numeric rating scale for pain (NRS pain), McGill pain questionnaire (MPQ), short-form McGill pain questionnaire (SF-MPQ), chronic pain grade scale (CpGs), short Form-36 bodily pain scale (SF-36 BPs), and measure of intermittent and constant osteoarthritis pain (ICOAP). Arthritis Care Res 2011;63 Suppl 11:S240-52.

44 Tate RL, Perdices M, Rosenkoetter U, et al. Revision of a method quality rating scale for single-case experimental designs and $\mathrm{N}$ - 
of- 1 trials: the 15 -item risk of bias in N-of- 1 trials (RoBiNT) scale. Neuropsychol Rehabil 2013;23:619-38.

45 Gatt JM, Burton KLO, Schofield PR, et al. The heritability of mental health and wellbeing defined using COMPAS-W, a new composite measure of wellbeing. Psychiatry Res 2014;219:204-13.

46 Spielberger CD, Gorsuch RL, Lushene R. Manual for the State-Trait anxiety inventory. Palo Alto: Consulting Psychologists' Press, 1970.

47 Beck AT, Ward CH, Mendelson M, et al. An inventory for measuring depression. Arch Gen Psychiatry 1961;4:561-71.

48 Hays RD, Stewart A. Sleep measures. In: Stewart AL, Ware JE, eds. Measuring functioning and well-being: the medical outcomes study approach. Duke University Press, 1992: 235-59.

49 Cloninger CR, Svrakic DM, Przybeck TR. A psychobiological model of temperament and character. Arch Gen Psychiatry 1993;50:975-90.

50 Lund AM. Measuring usability with the use questionnaire12. Usability interface 2001;8:3-6.

51 Widerström-Noga E, Biering-Sørensen F, Bryce TN, et al. The International spinal cord injury pain basic data set (version 2.0). Spinal Cord 2014;52:282.

52 Lane JD, Gast DL. Visual analysis in single case experimental design studies: brief review and guidelines. Neuropsychol Rehabil 2014;24:445-63.

53 Clanchy KM, Tweedy SM, Tate RL, et al. Evaluation of a novel intervention to improve physical activity for adults with whiplash associated disorders: protocol for a multiple-baseline, single case experimental study. Contemp Clin Trials Commun 2019;16:100455.

54 Tanious R, Onghena P. Randomized single-case experimental designs in healthcare research: what, why, and how? Healthcare 2019;7:143.
55 Parker RI, Vannest KJ, Davis JL. Effect size in single-case research: a review of nine nonoverlap techniques. Behav Modif 2011;35:303-22.

$56 \mathrm{Ma} \mathrm{H}-\mathrm{H}$. An alternative method for quantitative synthesis of singlesubject researches: percentage of data points exceeding the median. Behav Modif 2006;30:598-617.

57 Manolov R, Solanas A, Leiva D. Comparing "Visual" effect size indices for single-case designs. Methodology 2010;6:49-58.

58 Parker RI, Vannest KJ, Davis JL, et al. Combining nonoverlap and trend for single-case research: Tau-U. Behav Ther 2011;42:284-99.

59 Brossart DF, Laird VC, Armstrong TW. Interpreting Kendall's Tau and Tau- $U$ for single-case experimental designs. Cogent Psychology 2018:5:1518687.

60 Chen L-T, P-J W, Peng C-YJ. Accounting for baseline trends in intervention studies: methods, effect sizes, and software. Cogent Psychology 2019;6:1679941.

61 Vannest KJ, Parker RI, Gonen O. Single case research: web based calculators for SCR analysis. (Version 2.0 [Web-based application]. Texas, TX: Texas A\&M University: College Station, 2016. http://www. singlecaseresearch.org/calculators/tau-u

62 Stewart AL, Hays RD, Ware JE. The mos short-form general health survey. reliability and validity in a patient population. Med Care 1988:26:724-35.

63 Duff K. Evidence-based indicators of neuropsychological change in the individual patient: relevant concepts and methods. Arch Clin Neuropsychol 2012;27:248-61.

64 Maassen GH, Bossema E, Brand N. Reliable change and practice effects: outcomes of various indices compared. $J$ Clin Exp Neuropsychol 2009;31:339-52. 